\title{
Treatment with intralesional methotrexate injection in a patient with nail psoriasis
}

\section{Yesim Akpinar Kara}

\author{
Department of Dermatology, Liv Hospital, Ankara, Turkey
}

Corresponding author: Yesim Akpinar Kara, MD, E-mail: yesim_akpinar@yahoo.com

\begin{abstract}
Psoriasis vulgaris is an inflammatory skin disease involving the skin, nails, and joints. While nail involvement is observed in 70-80\% of patients with psoriasis, the rate of patients with isolated nail involvement is $5-10 \%$. Dystrophies arising in the nails in psoriasis affect the patient's quality of life, and local and systemic therapies may be used as treatment. Intralesional methotrexate or corticosteroid injection might be an option in the treatment of patients with the involvement of one nail or some nails or without the involvement of the skin and joints, due to the side effects of systemic and biological agents. Herein, we report a female patient with nail psoriasis resistant to a previously applied topical treatment, the efficacy of intralesional methotrexate without the use of a systemic antipsoriatic agent, and no progression of side effects.
\end{abstract}

Keywords: Psoriatic nails; Intralesional injection; Methotrexate

\section{INTRODUCTION}

Psoriasis is the most common skin disease affecting the nails. Nail changes may be associated with skin lesions while isolated nail psoriasis (1-5\%) without skin involvement may also appear [1]. Nail involvement in psoriasis is more common in the fingernails. Pitting, leukonychia, erythema of the lunula, which are among the nail findings of psoriasis, progress depending on the nail plate thickening and the matrix crumbling; salmon patches, splinter hemorrhage, subungual hyperkeratosis, and onycholysis are seen in the involvement of the nail bed [2].

Topical corticosteroids, intralesional injections of steroids or methotrexate, systemic and biological agents, and laser therapy are among the available treatments for nail psoriasis. Intralesional injections constitute another form of local treatment. Methotrexate (MTX), a folic acid analog, blocks the synthesis of deoxyribonucleic acid by binding to dihydrofolate reductase enzyme and produces an anti-proliferative and anti-inflammatory effect. It is known to be effective for psoriasis and other several skin diseases due to this effect [3].

Several studies on intralesional MTX injection in nail involvement in psoriasis have been reported [4-6]. During the treatment, maximum efficacy is obtained with a minimum dose of the drug, which is applied into the lesion. It might be considered that painful intralesional injection has restricted its use in the treatment of nail psoriasis, but it has been reported that the outcome of the treatment has been successful.

Herein, we present a patient with subungual hyperkeratosis, nail crumbling, and pitting on the nail, who was treated with intralesional methotrexate injection.

\section{CASE REPORT}

A 43-year-old female patient was admitted to the outpatient clinic complaining of squamous plaques on the scalp and behind both ears present for the previous five years as well as thickening and color changes in the fingernails for the previous eight months. The patient 
had used a topical corticosteroid and calcipotriol cream for psoriasis lesions on the scalp and behind both ears as well as a cream containing mometasone furoate for the nail lesions for three months, but these had not been effective. Erythematous, squamous plaques were detected in both the postauricular region and occipital region on the scalp on dermatological examination. Plate thickening and crumbling, subungual hyperkeratosis, pitting, and splinter hemorrhage were observed in the second fingernail of the right hand and in the fourth fingernail of the left hand (Fig. 1a) [7]. Laboratory investigations, including a complete blood count, sedimentation, C-reactive protein, liver and renal function tests, performed before and after treatment, showed no abnormalities. Fungi culture was negative in the samples taken from both nails. The Nail Psoriasis Severity Index (NAPSI) was determined to be at 16.

Before the treatment, written informed consent was taken. A $15 \mathrm{mg} / \mathrm{mL}(1.5 \mathrm{mg} / 0.1 \mathrm{~mL})$ dose of MTX was injected subepidermally under the matrix on both lateral sides of the proximal nail fold through a 30 -gauge syringe at a dose of $0.1 \mathrm{~mL}$, after both of the two proximal phalanges and nail regions were occluded through topical mixture of lidocaine $2.5 \%$ and prilocaine for one hour. The injection was repeated for every affected nail every four weeks for six consecutive months. No side effects were observed. A significant improvement was observed in nail dystrophy within six months of follow-up (Fig. 1b) [7]. The patient is currently on follow-up and no clinical relapse was observed after two years.

\section{DISCUSSION}

Nail psoriasis is known to be resistant to numerous treatment methods more than cutaneous psoriasis, including to potent biological agents. The number of nail involvements, the effect on the quality of life, and arthritis are effective in determining the proper treatment method [2]. With more than three nail involvements, systemic treatment is recommended in the literature. Topical or intralesional injection

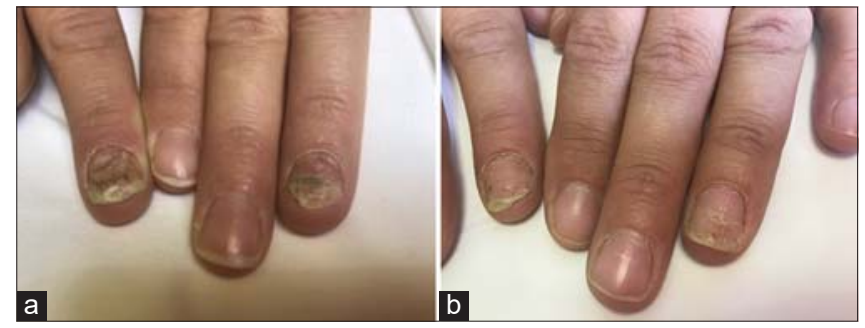

Figure 1: (a) Nail dystrophy in the fingernails before the injection; (b) six months of follow-up after treatment. treatments may be used if less than three nails are involved. If nail dystrophy is limited to the matrix, it is recommended that a topical steroid and topical vitamin $\mathrm{D}$ analogs, or topical $0.1 \%$ tacrolimus ointment with nail bed involvement, intralesional injection may be added to the treatment [8].

Intralesional steroid injection is effective especially if there is no response from topical treatment in isolated nail involvement. The possible complications of this treatment are atrophy of the terminal phalanx bone, extensor tendon rupture, and epidermoid inclusion cysts [2]. Several publications in the literature on intralesional injection of methotrexate for nail psoriasis may be found. In a study reported by Saraçoğlu et al., patients with nail psoriasis were treated with intralesional MTX to the lateral points of the proximal nail fold once a week for six weeks, and no recurrence was observed during a two-year follow-up period [4].

Mokni and Duarte reported progressive improvement in nail dystrophy with intralesional MTX injection in nail psoriasis $[9,10]$. Mittal et al. reported that intralesional methotrexate and corticosteroid injections show similar efficacy in nail psoriasis. They also reported that intramaterial methotrexate provided the biggest improvement with minimal side effects [5-7].

Jiaravuthisan et al. recommended the use of topical corticosteroids, tazarotene, calcipotriol cream, or intralesional steroid injection and cyclosporine if there is a small number of nail involvements as well as the use of a retinoid and infliximab in systemic treatment if there is a large number of nail involvement in an algorithm that they produced for the treatment of nail psoriasis [6].

Nail lesions, which progress depending on the psoriasis, affect the patient cosmetically and damage their quality of life. Intralesional MTX injection may be effective due to the fact that it shows a smaller number of side effects than systemic agents, and the outcome of treatment is successful if a small number of nails are affected in the psoriasis. Studies with randomized controls with multiple cases need to be conducted in order to confirm the efficacy of the treatment.

\section{ACKNOWLEDGMENTS}

The figures used in this article are original and belong to the author. The chapter in the book where these pictures were published was cited in the reference section. 


\section{Consent}

The examination of the patient was conducted according to the principles of the Declaration of Helsinki.

The authors certify that they have obtained all appropriate patient consent forms, in which the patients gave their consent for images and other clinical information to be included in the journal. The patients understand that their names and initials will not be published and due effort will be made to conceal their identity, but that anonymity cannot be guaranteed.

\section{REFERENCES}

1. Salomon J, Szepietowski JC, Proniewicz A. Psoriatic nails: A prospective clinical study. J Cutan Med Surg. 2003;7:317-21.

2. Haneke E. Nail psoriasis: Clinical features, pathogenesis, differential diagnoses, and management. Psoriasis (Auckl). 2017;16:51-63.

3. Chan ES, Cronstein BN. Methotrexate - How does it really work? Nat Rev Rheumatol. 2010;6:175-8.

4. Sarıcaoglu $\mathrm{H}, \mathrm{Oz}$ A, Turan $\mathrm{H}$. Nail psoriasis successfully treated with intralesional methotrexate: Case Rep Dermatol. 2011; 222:5-7.

5. Mittal J, Mahajan BB. Intramatricial injections for nail psoriasis: An open-label comparative study of triamcinolone, methotrexate, and cyclosporine. Indian J Dermatol Venereol Leprol. 2018;84: 419-23.

6. Jiaravuthisan MM, Sasseville D, Vender RB, Murphy F, Muhn CY. Psoriasis of the nail: Anatomy, pathology, clinical presentation, and a review of the literature on therapy. J Am Acad Dermatol. 2007;57:1-27.

7. Kara YA. Chapter the etiology, pathophysiology, differential diagnosis, clinical findings and treatment of nail psoriais.1n: shahin aghaei, tailored treatments in psoriatic patients. $1^{\text {th }}$ ed.London, PA: Intechopen; 2019.

8. Rigopoulos D, Baran R, Chiheb S, Daniel CR, Di Chiacchio N, Gregoriou S, et al. Recommendations for the definition, evaluation, and treatment of nail psoriasis in adult patients with no or mild skin psoriasis: A dermatologist and nail expert group consensus. J Am Acad Dermatol. 2019;81:228-40.

9. Mokni S, Ameur K, Ghariani N, Sriha B, Belajouza C, Denguezli M, et al. A case of nail psoriasis successfully treated with intralesional methotrexate. Dermatol Ther (Heidelb). 2018;8:647-51.

10. Duarte AA, Carneiro GP, Murari CM, Jesus LCB. Nail psoriasis treated with intralesional methotrexate infiltration. An Bras Dermatol. 2019;17:94:491-2.

Copyright by Yesim Akpinar Kara. This is an open access article distributed under the terms of the Creative Commons Attribution License, which permits unrestricted use, distribution, and reproduction in any medium, provided the original author and source are credited.

Source of Support: Nil, Conflict of Interest: None declared. 